\title{
Attentional spread in deaf and hearing participants: Face and object distractor processing under perceptual load
}

\author{
Nadine Hauthal • Markus F. Neumann • \\ Stefan R. Schweinberger
}

Published online: 26 May 2012

(C) Psychonomic Society, Inc. 2012

\begin{abstract}
The case of human deafness constitutes a unique opportunity to examine possible consequences for perceptual processing due to altered sensory experiences. We tested whether deaf - in contrast to hearing - individuals are more susceptible to visual distraction from peripheral than from central face versus object stimuli. The participants were required to classify the gender of a target male or female symbol presented either alone (low perceptual load) or together with three filler symbols (high perceptual load), while ignoring gender-congruent or -incongruent face versus object distractors presented at central or peripheral positions. The gender classifications were affected by distractor gender under low, but not under high, perceptual load in hearing participants. In contrast, the responses of deaf participants were similarly influenced by distractor gender under both levels of perceptual load. There was no evidence for generally enhanced attention to the visual periphery in deaf individuals. Our results indicate that auditory
\end{abstract}

Electronic supplementary material The online version of this article (doi:10.3758/s13414-012-0320-1) contains supplementary material, which is available to authorized users.

\section{N. Hauthal $(\square)$}

Department of Psychology, Neuropsychology Lab,

Carl von Ossietzky University of Oldenburg,

Oldenburg, Germany

e-mail: nadine.hauthal@uni-oldenburg.de

N. Hauthal • M. F. Neumann · S. R. Schweinberger

Department of General Psychology and Cognitive Neuroscience, Friedrich Schiller University of Jena,

Jena, Germany

S. R. Schweinberger

DFG Research Unit Person Perception,

Friedrich Schiller University of Jena,

Jena, Germany deprivation may result in enhanced attentional capacities under high perceptual load.

Keywords Spatial vision - Selective attention · Face perception $\cdot$ Deafness $\cdot$ Auditory deprivation $\cdot$ Perceptual load $\cdot$ Periphery

A central question when investigating deafness is whether deprivation in the auditory domain causes alterations in the functioning of the remaining senses. Due to the absence of auditory information, deaf individuals must rely heavily on the visual sense to obtain information from the environment. However, it has been shown that general visual abilities are not altered in deaf individuals (Brozinsky \& Bavelier, 2004; Finney \& Dobkins, 2001; Poizner \& Tallal, 1987). Bavelier, Dye, and Hauser (2006) proposed that an advantage in visual processing for deaf individuals might occur for stimuli that are presented in the periphery and/or in motion, especially under attentionally demanding conditions.

Accordingly, individuals with profound hearing loss have detected static and moving peripheral targets faster and at larger eccentricities than did hearing individuals (e.g., Loke \& Song, 1991; Stevens \& Neville, 2006). Enhanced reactivity to visual stimuli in deaf participants has also been reported to occur for both central and peripheral stimuli (Bottari, Nava, Ley, \& Pavani, 2010; see Pavani \& Bottari, 2011, for a recent review). Remarkably, deaf individuals exhibit larger visual fields than do normal-hearing controls (Buckley, Codina, Bhardwaj, \& Pascalis, 2010; Codina et al., 2011; Stevens \& Neville, 2006). Monitoring moving stimuli in the visual periphery produced higher activation in motion-related areas (middle temporal and medial superior temporal area) in deaf than in hearing participants (Bavelier et al., 2001; Bavelier et al., 2000). Moreover, deaf individuals exhibited larger interference effects from 
irrelevant peripheral distractors while classifying central targets (Dye, Baril, \& Bavelier, 2007; Sladen, Tharpe, Ashmead, Grantham, \& Chun, 2005). Such differences between deaf and hearing individuals in allocating visual spatial attention seem to be a result of auditory deprivation, and not of sign language use (Dye, Hauser, \& Bavelier, 2009; Neville \& Lawson, 1987c; Proksch \& Bavelier, 2002). Similar phenomena were observed in blind individuals who showed better sound source localization abilities, especially in peripheral auditory space (Fieger, Röder, Teder-Sälejärvi, Hillyard, \& Neville, 2006; Röder et al., 1999).

Considering that enhanced visual processing in deaf individuals is supposed to occur especially under attentionally demanding conditions (Bavelier et al., 2006), we directly manipulated attention according to the perceptual load theory (Lavie, 1995, 2005). The perceptual load theory assumes that individuals have access to a specific amount of attentional capacity. A crucial assumption of the perceptual load theory is that voluntary withholding of attentional capacity is impossible, meaning that attentional capacity does not remain free. Instead, if the processing of relevant information does not require all of the available resources (leaving free capacity) under low perceptual load, additional, irrelevant information will be automatically processed until the capacity limit is reached. However, increasing perceptual load will eventually eliminate irrelevant distractor processing, because relevant information already requires all available attentional capacity.

Proksch and Bavelier (2002) compared the attentional resources of deaf and hearing individuals. Participants were asked to identify a target square or diamond among up to five nontarget fillers, arranged in a circle. Perceptual load was gradually manipulated by presenting zero (target only), one, three, or five irrelevant fillers. Additionally, distractors were simultaneously displayed either peripherally (outside of the circle) or centrally (inside of the circle) and were congruent to the target (identical stimulus), incongruent (respective other target stimulus), or neutral (an elongated circle without associated response). Hearing participants revealed larger interference from central incongruent distractors. In contrast, larger interference effects were caused by peripheral distractors in deaf participants. This may be explained by a relative shift in the distribution of attention in deaf individuals toward more peripheral spatial locations.

In the present study, we asked whether the previously described advantage for deaf individuals in processing peripheral stimuli (Proksch \& Bavelier, 2002) also applies to more complex and meaningful stimuli. We were specifically interested in distractor interference effects from everyday objects and human faces. In particular, faces are socially and biologically extremely relevant, and they attract attention more than objects do (Langton, Law, Burton, \& Schweinberger, 2008; Ro, Russell, \& Lavie, 2001).

We used a modified version of the distractor interference paradigm by Proksch and Bavelier (2002). Male and female faces, as well as typical male and female objects, served as distractors. Participants made speeded gender classifications to a male or a female symbol target presented either alonethat is, under low perceptual load-or along with three nontargets - that is, under high perceptual load. Gender congruency between the target symbol and the distractor face/object was manipulated. A congruency effect, caused by differences in participants' performance with gendercongruent and -incongruent combinations, therefore reflects the extent of distractor processing.

Here, we asked whether hearing and deaf individuals' processing of faces versus objects is similarly affected by imposing perceptual load and whether differential patterns would occur for the two groups with respect to central versus peripheral distractor position. Preferential face processing may persist in all participants under high perceptual load, when faces are presented either peripherally or centrally (Lavie, Ro, \& Russell, 2003; Neumann, Mohamed, \& Schweinberger, 2011). However, please note that auditory deprivation may impose special demands on face processing. For example, lip reading plays an important role in the communication of deaf with hearing individuals. Furthermore, expressive and articulatory facial motion provides important information in sign language use. Deaf people have been reported to fixate mostly on the face of the signer (perhaps, remarkably, with the upper half of the face receiving more fixation time than the lower half), whereas peripheral vision is used to process information from larger rapid movements of the signer (Muir \& Richardson, 2005). Accordingly, one might expect greater interference from distractor faces for deaf than for hearing individuals.

Furthermore, we tested the prediction that deaf participants would be more susceptible to incongruent distraction in the visual periphery. Accordingly, individuals with profound hearing loss should display larger distraction from faces and objects in the visual periphery than would hearing participants. In contrast, distraction from central items may be expected to be either comparable in both groups (judging from the fact that no group differences were found for central target discrimination-see Bottari et al., 2010; Neville \& Lawson, 1987a, 1987b-or for central target detection-see Loke \& Song, 1991; but see also Bottari et al., 2010) or should even be more pronounced for hearing participants (see Chen, He, Chen, Jin, \& Mo, 2010; Proksch \& Bavelier, 2002). Additionally, interference was expected to be modulated by perceptual load. As described in more detail above, the perceptual-load theory (Lavie, 1995, 2005) predicts that distracting effects will be generally larger under low than under high perceptual load, because high-load 
displays consume most or all of the attentional capacity available, thus leaving little or no free capacity for distractor processing.

\section{Method \\ Participants}

A group of 18 deaf individuals was tested. All but one reported suffering from profound binaural hearing loss, which led to the exclusion of the single participant. Another deaf participant was excluded due to a cataract. The mean age of the remaining 16 deaf participants was 45.5 years (range 29-57; nine female, seven male). Eight of the participants were congenitally deaf; the remaining eight had lost their hearing before age six. All of the deaf participants were fluent users of German Sign Language. An additional 19 hearing controls were tested; three of these were excluded due to suffering from tinnitus. The remaining 16 controls were each assigned to a deaf participant of the same gender and age ( \pm 2 years). The mean age of the 16 hearing controls was 44.9 years (range 28-58; nine female, seven male). All of the participants were Caucasian and lived in the same geographical region in Germany. The average durations of school attendance were 9.9 years $(S D=0.5)$ for deaf individuals and 11.4 years $(S D=1.4)$ for hearing individuals. It has to be noted that a precise control for educational background is difficult to create because of the structural particularities of deaf education. All deaf and hearing participants reported normal or corrected-tonormal vision, and neither group reported having played any action video games over the last 12 months. All participants gave informed consent and were compensated for their participation.

\section{Design and stimuli}

Two common gender symbols (one male and one female silhouette) served as the targets; three nongender symbols were used as filler figures (see Fig. 1). The target size was $1.6 \times 2.8$ of visual angle (VA) at a viewing distance of $90 \mathrm{~cm}$. The filler figures comprised shapes similar to those of the targets and were matched in terms of size and luminance. Additionally, filler circles of $0.5^{\circ} \mathrm{VA}$ were used.

The distractors (sized $1.4^{\circ} \times 1.8^{\circ} \mathrm{VA}$ ) were 16 grayscale photographs of unfamiliar Caucasian faces (eight male and eight female) taken from the CAL/PAL face database (Minear \& Park, 2004), and 16 "gender-typical" objects (for a comparable approach, see Brebner \& Macrae, 2008). The gender typicality of 43 objects was rated by 27 hearing new Caucasian participants (mean age $=31.3$ years, range 18-71 years; 15 female, 12 male). The nine objects with the highest female and male ratings, respectively, were selected (see the supplementary materials). One image per category was used in practice trials only. All of the stimuli were presented on a black background. Although the image attributes were somewhat more variable for the objects than for the faces, the mean luminances of the distractor categories were kept at comparable levels. The mean luminances of both distractor categories (measured by averaging the grayscale value of each pixel, ranging from 0 [black] to 255 [white] using Adobe Photoshop CS5) did not differ significantly (faces, $M=118.8, S E M=4.1$; objects, $M=$ 125.9, SEM = 12.7), $t(15)=-0.52, p>.60$.

Target and fillers were arranged in an imaginary square with each stimulus positioned in one of the four corners (see Fig. 1). For high-perceptual-load trials, one target symbol was presented together with three different filler symbols, while for low-perceptual-load trials, a target symbol was combined with three identical filler circles. The target and filler positions were assigned randomly. A distractor could be presented either in the center of the stimulus display ( $50 \%$ of the trials) or in the periphery $(25 \%$ each for the left and right sides of the stimulus display). In one half of the trials, the distractor was a photograph of a face, in the other half it was a photograph of an object. In the case of a peripheral distractor, eccentricity was $4.4^{\circ} \mathrm{VA}$. The target and fillers were presented at an eccentricity of $2.2^{\circ}$ VA. In $50 \%$ of the trials apiece, the target symbol and distractor face/object were gender-congruent (male/male or female/ female) or gender-incongruent (male/female or female/ male).

\section{Procedure}

Participants were seated $90 \mathrm{~cm}$ in front of the monitor in a dimly lit room. Distance was held constant by using a chinrest. A trial began with a fixation square, the corners of which indicated the potential target locations (cf. Fig. 1 for examples of the trial sequences). A fixation cross was not used in this study, in order to avoid cueing of the central distractor (for a similar approach, see Beck \& Lavie, 2005). The fixation square appeared for $1,000 \mathrm{~ms}$ and was immediately replaced by the display containing target symbol, fillers, and distractor. This was presented for $200 \mathrm{~ms}$ and followed by a blank screen for $1,800 \mathrm{~ms}$, during which time participants responded via button presses. The task was to decide whether the target symbol was male or female by pressing a marked "a" or "l" key on a standard keyboard using the index fingers. The key assignments were counterbalanced across participants, who were instructed to classify the target as quickly and accurately as possible while ignoring the distractor. Hearing participants got written instructions on the screen, whereas deaf participants received the same instructions additionally via sign language. High- and 
Fig. 1 Top row: A lowperceptual-load trial. The peripheral (female) distractor face is incongruent with the male target symbol. Bottom row: A high-perceptual-load trial. Here, a central (female) object distractor is congruent with the female target symbol presented among nontarget symbols

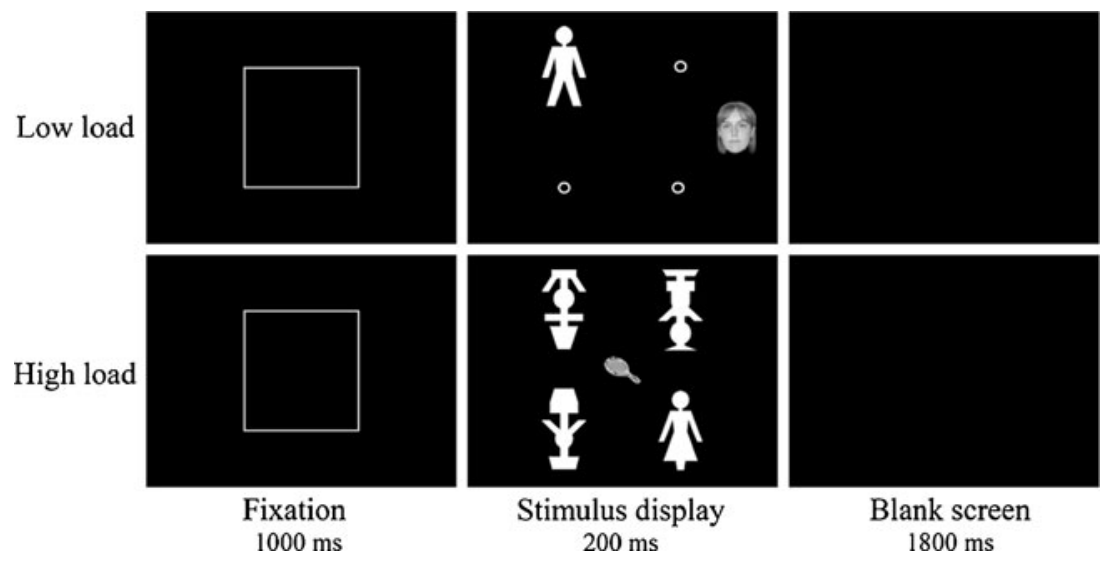

low-perceptual-load conditions were presented in two blocks separated by a break. Each block consisted of 256 trials, allowing for a break after every 64 trials. The order of blocks was counterbalanced across participants.

During four practice blocks, including a total of 56 trials, written feedback was given for correct and incorrect, as well as for absent, responses. During practice, the display complexity was gradually increased, starting with low perceptual load without a distractor (eight trials), high perceptual load without a distractor (16 trials), low perceptual load with a distractor (16 trials), and high perceptual load with a distractor (16 trials). The whole experiment lasted about $40 \mathrm{~min}$.

\section{Results}

Analyses of variance (ANOVAs) were carried out with repeated measures using the within-subject factors Perceptual Load (low vs. high), Distractor Position (center vs. periphery), Distractor Type (face vs. object), and Congruency of the target and distractor (congruent vs. incongruent), as well as the between-subjects factor Group (deaf vs. hearing). The mean response times (RTs) and error rates were analyzed (see Table 1). For analyses of the RTs, trials with an incorrect response or without any response were excluded $(6.2 \%)$. To avoid outliers, responses slower than two standard deviations above the mean $(M=704.5 \mathrm{~ms}, S D=$ $182.5 \mathrm{~ms})$ were excluded $(4.0 \%)$.

\section{Response times}

The ANOVA on RTs revealed a main effect of perceptual load, $F(1,30)=38.65, p<.001$. Accordingly, participants responded faster under low $(M=653.2 \mathrm{~ms})$ than under high $(M=712.9 \mathrm{~ms})$ load. A main effect of distractor position, $F$ $(1,30)=17.05, p<.001$, indicated that RTs were slower when a distractor was presented in the center $(M=$ $686.9 \mathrm{~ms})$ rather than in the periphery $(M=679.1 \mathrm{~ms})$.
Responses to the target symbol were slower when it was accompanied by a face $(M=686.5 \mathrm{~ms})$ rather than by an object $(M=679.6 \mathrm{~ms})$ distractor, $F(1,30)=10.36, p<.01$. A main effect of congruency was also found, $F(1,30)=$ $18.64, p<.001$, with slower responses to incongruent $(M=686.4 \mathrm{~ms})$ than to congruent $(M=679.7 \mathrm{~ms})$ trials. In addition, the main effect of group approached significance, $F(1,30)=4.10, p=.052$ : Hearing participants tended to respond faster than deaf participants $(M \mathrm{~s}=659.4$ vs. $706.7 \mathrm{~ms}$ ).

Furthermore, the interaction between distractor position and distractor type was significant, $F(1,30)=8.47, p<.01$ (see Fig. 2). Post-hoc pairwise comparisons revealed that for central distractors, target classification was slower with a face $(M=693.1 \mathrm{~ms})$ than with an object $(M=680.7 \mathrm{~ms})$ being presented, $t(31)=4.33, p<.001$. No such difference was observed for peripheral distractors $(M s=679.8$ vs. $678.5 \mathrm{~ms}), t(31)=0.48, p>.60$.

Importantly, the three-way interaction between group, distractor position, and congruency was not significant, $F$ $(1,30)=0.16, p>.60$ (see Fig. 3), nor was any higher-order interaction including group and distractor position as a factors, all $p s>.13$.

However, the interaction between group, perceptual load, and congruency was significant, $F(1,30)=7.21, p<.05$ (see Fig. 4). Hence, 2 (perceptual load) $\times 2$ (congruency) ANOVAs were conducted separately for each group. For hearing participants, the main effects of perceptual load, $F$ $(1,15)=34.48, p<.001$, and congruency, $F(1,15)=13.59$, $p<.01$, were significant, as well as the interaction of both factors, $F(1,15)=5.48, p<.05$. Subsequent paired-samples $t$-tests revealed faster responses for congruent $(M=$ $620.5 \mathrm{~ms})$ than for incongruent $(M=634.8 \mathrm{~ms})$ trials under low load, $t(15)=4.76, p<.001$. No congruency effect was observed under high load $(M s=689.9$ vs. $692.5 \mathrm{~ms}), t(15)=$ $0.71, p>.40)$. For deaf participants, there were significant main effects of perceptual load, $F(1,15)=12.38, p<.01$, with faster responses under low $(M=678.8 \mathrm{~ms})$ than under high $(M=734.6 \mathrm{~ms})$ load, and of congruency, $F(1,15)=$ 
Table 1 Response times (in milliseconds) \pm standard errors of the means, along with error rates (in parentheses) in deaf and hearing participants, for all combinations of distractor types (faces vs. objects) and distractor positions (central vs. peripheral) under both load conditions (high load [HL] vs. low load [LL]) and separately for gendercongruent and gender-incongruent target-distractor combinations

\begin{tabular}{|c|c|c|c|c|c|c|c|}
\hline & & \multicolumn{3}{|l|}{ Central } & \multicolumn{3}{|l|}{ Peripheral } \\
\hline & & Incongruent & Congruent & $\mathrm{I}-\mathrm{C}$ & Incongruent & Congruent & $\mathrm{I}-\mathrm{C}$ \\
\hline \multicolumn{8}{|l|}{ Deaf } \\
\hline \multirow[t]{2}{*}{ Faces } & LL & $696.2 \pm 17.9(6.9)$ & $678.6 \pm 17.7(3.8)$ & $17.6 \pm 7.6(3.1)$ & $680.3 \pm 19.3$ & $668.1 \pm 16.9(5.0)$ & $12.2 \pm 8.8(-0.3)$ \\
\hline & HL & $748.5 \pm 20.2(9.6)$ & $742.3 \pm 18.3(7.6)$ & $6.3 \pm 9.8(2.1)$ & $737.1 \pm 19.4(6.2)$ & $727.0 \pm 21.5(9.1)$ & $10.2 \pm 5.7(-2.9)$ \\
\hline \multirow[t]{2}{*}{ Objects } & LL & $675.9 \pm 16.2(5.3)$ & $683.0 \pm 16.6(4.6)$ & $-7.1 \pm 5.2(0.7)$ & $668.6 \pm 18.1(5.4)$ & $679.6 \pm 17.2(3.7)$ & $-11.0 \pm 7.9(1.7)$ \\
\hline & HL & $733.8 \pm 18.4(7.9)$ & $724.8 \pm 20.5(6.5)$ & $9.0 \pm 4.9(1.4)$ & $732.8 \pm 18.3(6.7)$ & $730.2 \pm 18.5(5.6)$ & $2.7 \pm 7.4(1.2)$ \\
\hline \multicolumn{8}{|l|}{ Hearing } \\
\hline \multirow[t]{2}{*}{ Faces } & LL & $645.6 \pm 17.9(2.7)$ & $634.2 \pm 17.7(3.3)$ & $11.4 \pm 8.6(-0.6)$ & $629.1 \pm 19.3$ & $615.5 \pm 16.9(2.8)$ & $13.6 \pm 6.3(-1.5)$ \\
\hline & HL & $703.8 \pm 20.2(6.8)$ & $695.7 \pm 18.3(6.8)$ & $8.2 \pm 8.0(0.0)$ & $689.4 \pm 19.4(5.6)$ & $692.1 \pm 21.5(5.7)$ & $-2.7 \pm 6.6(-0.1)$ \\
\hline \multirow[t]{2}{*}{ Objects } & LL & $636.8 \pm 16.2(3.2)$ & $618.9 \pm 16.6(2.4)$ & $17.9 \pm 6.2(0.7)$ & $627.6 \pm 18.1$ & $613.2 \pm 17.2(2.5)$ & $14.4 \pm 5.1(0.5)$ \\
\hline & $\mathrm{HL}$ & $684.9 \pm 18.4(5.2)$ & $687.9 \pm 20.5(6.3)$ & $-2.9 \pm 7.2(-1.1)$ & $691.9 \pm 18.3(2.2)$ & $683.9 \pm 18.5(3.2)$ & $8.0 \pm 8.7(-1.0)$ \\
\hline
\end{tabular}

"I - C" refers to the congruency effect - that is, incongruent minus congruent conditions

$5.59, p<.05$, indicating that participants responded faster in congruent $(M=704.2 \mathrm{~ms})$ than in incongruent $(M=$ $709.2 \mathrm{~ms}$ ) trials. The interaction between perceptual load and congruency was not significant for deaf participants, $F$ $(1,15)=1.75, p>.20$.

\section{Error rates}

The corresponding ANOVA on error rates revealed a main effect of perceptual load, $F(1,30)=10.84, p<.01$ : Participants made more errors under high $(M=8.1 \%)$ than under low $(M=4.2 \%)$ load. The main effect of distractor position also reached significance, $F(1,30)=4.35, p<.05$, with higher error rates when central $(M=6.6 \%)$ than when peripheral $(M=5.7 \%)$ distractors were presented. Furthermore, there was a main effect of distractor type, $F(1,30)=$ $7.65, p<.05$, with higher error rates on trials with a face $(M=6.7 \%)$ than with an object $(M=5.6 \%)$ distractor

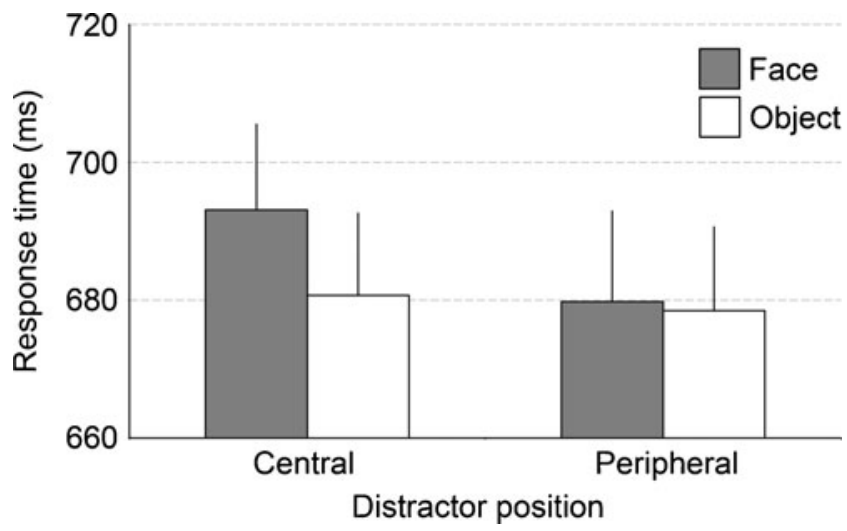

Fig. 2 Mean response times (in milliseconds) for central and peripheral distractor positions as a function of distractor type. Error bars represent the standard errors of the means accompanying the target. Deaf and hearing participants did not differ significantly in their overall performance $(M \mathrm{~s}=$ $7.1 \%$ and $5.2 \%$, respectively), $F(1,30)=1.41, p>.20$.

The interaction between perceptual load and distractor type was significant, $F(1,30)=5.68, p<.05$. Pairedsamples $t$-tests showed that under high load, participants made more errors with a face $(M=9.2 \%)$ than with an object $(M=7.1 \%)$ distractor occurring in the stimulus display, $t(31)=3.02, p<.01$. No such difference was observed under low load $(M \mathrm{~s}=4.2 \%$ vs. $4.1 \%), t(31)=$ $0.18, p>.80$.

Furthermore, the interaction between group and congruency reached significance, $F(1,30)=4.24, p<.05$. Numerically, both participant groups were more error prone in incongruent than in congruent trials, but the difference was more pronounced in the deaf group. However, post-hoc comparisons conducted separately for both groups were not significant [deaf, $t(15)=-1.66, p=.12(M \mathrm{~s}=7.8 \%$

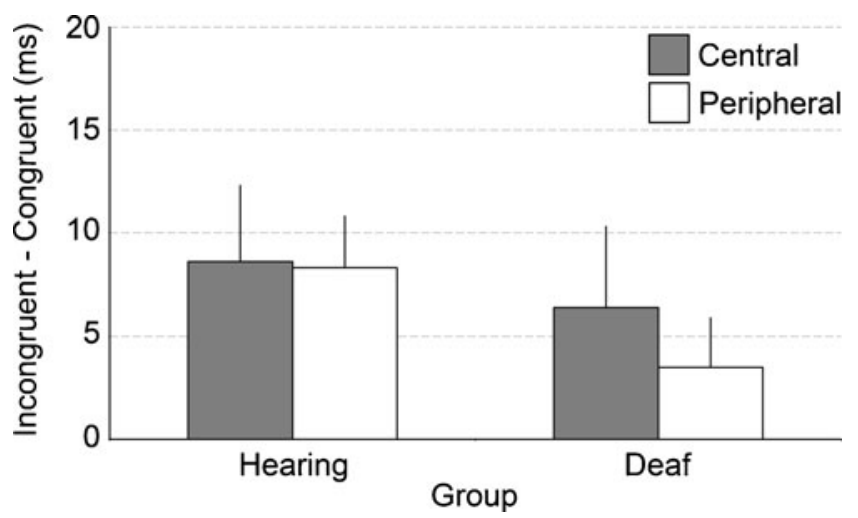

Fig. 3 Congruency effects (incongruent minus congruent, in milliseconds) for each group as a function of distractor position. Error bars represent the standard errors of the means 


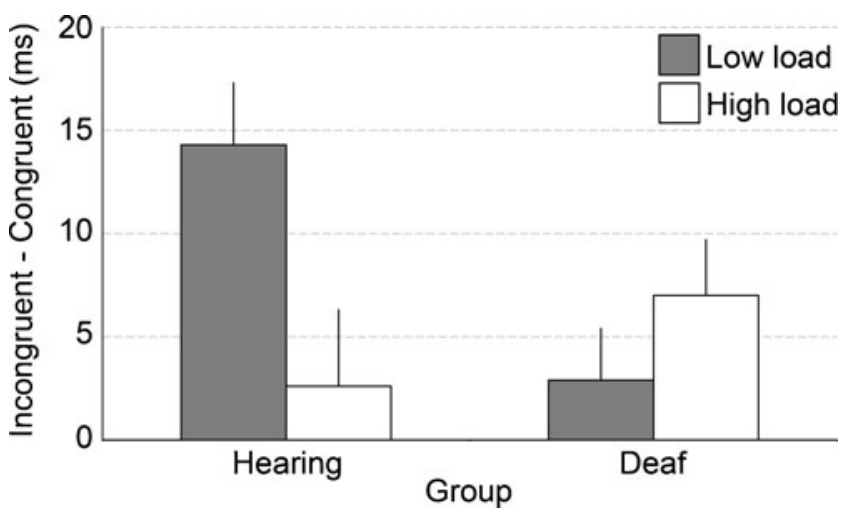

Fig. 4 Congruency effects (incongruent minus congruent, in milliseconds) for each group as a function of perceptual load. Error bars represent the standard errors of the means

vs. $6.4 \%)$; hearing, $t(15)=1.27, p=.22(M \mathrm{~s}=5.5 \%$ vs. $4.9 \%)]$.

\section{Discussion}

Several previous studies have reported a different distribution of spatial attention in individuals who experienced a profound hearing loss, as compared to normal-hearing individuals. For example, enhanced processing of stimuli in the visual periphery occurred in deaf individuals (Chen, Zhang, \& Zhou, 2006; Loke \& Song, 1991; Neville \& Lawson, 1987a, 1987b; Stevens \& Neville, 2006), and they were also shown to be more susceptible to peripheral distraction (Chen et al., 2010; Dye et al., 2007; Proksch \& Bavelier, 2002; Sladen et al., 2005). In contrast, greater susceptibility to central distraction was found in hearing individuals (Chen et al., 2010; Proksch \& Bavelier, 2002). Here, we examined the processing of irrelevant distractor faces and objects in hearing and deaf participants while manipulating distractor position and perceptual load. Overall, deaf individuals tended to respond more slowly than did hearing participants, which is in line with several previous studies (Dye et al., 2007; Parasnis \& Samar, 1985; Sladen et al., 2005; but see Proksch \& Bavelier, 2002). However, the deaf and hearing participants did not differ in accuracy, suggesting that group characteristics (such as educational background) did not result in overall differences in task performance, and thus are unlikely to explain the specific pattern of effects observed. As a potential limitation, while we instructed our participants to keep their eyes at the center of the display, we did not control for eye movements (see also Proksch \& Bavelier, 2002). A recent study indicated that, relative to hearing participants, deaf participants exhibited differentially faster saccadic responses in a prosaccade, as opposed to an antisaccade, task, particularly when the task was repeated relative to the preceding trial (Bottari, Valsecchi, \& Pavani,
2012). However, the mean latencies for both types of saccades peaked between around 260 and $350 \mathrm{~ms}$, respectively, in that study, well beyond the $200 \mathrm{~ms}$ that constituted the display offset in the present study. Overall, it thus seems unlikely that eye movements - group differences in saccadic latencies, in particular-influenced the present pattern of results.

Congruency effects and perceptual load

Our manipulation of perceptual load was successful, as seen by slower responses and larger error rates to targets under high than under low load (cf. Beck \& Lavie, 2005; Lavie, 1995). Importantly, a congruency effect occurred, such that gender-incongruent combinations of target and distractor led to slower responses than did gender-congruent combinations. This indicates that participants processed distractor gender, even though it was not relevant for the task. More specifically, both face and object distractor gender in this study interfered with gender decisions regarding the target symbols, in line with previous observations for both faces (Neumann, Schweinberger, Wiese, \& Burton, 2007) and objects (Brebner \& Macrae, 2008). We note that-although significant - the congruency effect in the present study was relatively small when compared to those in other studies using face distractors (Brebner \& Macrae, 2008; Lavie et al., 2003; Neumann et al., 2007). One possibility is that the relatively small size of the congruency effect in this study relates to the lack of a sharp contrast between low- and highload trials, due to the blocked presentation.

Most importantly, congruency effects in the present study were differentially affected by perceptual load in both groups, as suggested by the significant three-way interaction of group, perceptual load, and congruency. In line with previous research, hearing participants showed a significant congruency effect under low, but not under high load (Lavie, 1995; Lavie et al., 2003). By contrast, the congruency effect for deaf participants was significant and remained clear under conditions of high perceptual load. Accordingly, the deaf participants processed distractors even under high load, whereas no such distractor processing occurred for hearing individuals, likely due to the exhaustion of free capacity. Actually, deaf participants showed a numerically, but not significantly, larger congruency effect under high than under low load. This is in contrast to perceptual load theory (Lavie, 1995, 2005), which assumes that more attentional capacity is free under low than under high load, and therefore predicts a more pronounced congruency effect under low than under high load.

While novel, the findings under high load are reminiscent of those reported in an earlier study by Parasnis and Samar (1985), in which participants were asked to detect a parafoveal circle with or without task-irrelevant foveal 
information being presented. A central arrow cued the target position. Critically, in the case of an invalid cue, deaf individuals were more effective than hearing controls in redirecting attention to the target location, but only when the target was presented with additional foveal informationthat is, under high perceptual load. Similarly, Dye, Hauser, and Bavelier (2009) reported that deaf participants localized a peripheral target star among irrelevant squares faster than did hearing participants when they simultaneously had to classify another central target. In sum, and similar to the present study, hearing participants may already have exceeded their attentional capacity limit through additional load, whereas deaf participants seemed to retain free capacity, allowing fast redirection of attention (Parasnis \& Samar, 1985) or fast localization (Dye et al., 2009). We suggest that deaf individuals exhibit increased visual attentional capacity, as compared to their hearing counterparts.

\section{Congruency effects and distractor position}

Previous reports have suggested more widespread attention to peripheral locations in deaf than in hearing participants (e.g., Dye et al., 2007, Sladen et al., 2005; Stevens \& Neville, 2006). Accordingly, distractor position was assumed to differentially affect congruency effects in the two participant groups in the present study. For deaf participants, larger congruency effects were expected from peripheral distractors, whereas central distractors should cause either comparable congruency effects in both groups or more pronounced effects for hearing participants. However, the present results indicate that deaf and hearing participants showed comparable congruency effects from central and peripheral distractor positions, suggesting similar distributions of attention across the visual field. The dichotomy of central versus peripheral visual processing may actually be too unspecific, and a "gradient view" of selective attention (cf. Downing \& Pinker, 1985) may more appropriately capture the effects of attention across the visual field (for a recent review, see Pavani \& Bottari, 2011).

Despite using distractor eccentricity similar to that in the present study, Proksch and Bavelier (2002) additionally accounted for cortical magnification - that is, the fact that stimuli at fixation are perceived with higher visual acuityby increasing the size of peripheral distractors (cf. Rovamo \& Virsu, 1979). The absence of enhanced processing of peripheral distractors for deaf participants in the present study could thus be due to the fact that cortical magnification was not controlled for. However, the congruency effects did not differ numerically between central and peripheral distractor locations in hearing participants, suggesting that cortical magnification did not play a crucial role here. Furthermore, neural changes in the retinal structures of deaf participants suggested a greater retinal ganglion cell number and were related to an enhanced peripheral vision (Codina et al., 2011), which makes the application of the corticalmagnification factor (Rovamo \& Virsu, 1979) to deaf individuals more complex.

We believe that our use of photographs of faces and objects instead of rather meaningless material (Armstrong, Neville, Hillyard, \& Mitchell, 2002; Loke \& Song, 1991; Neville \& Lawson, 1987a, 1987b; Proksch \& Bavelier, 2002; Stevens \& Neville, 2006; but see Bottari et al., 2008) may well have resulted in the present pattern. One could speculate whether, for deaf participants, widespread attention is a "default" mode of attention allocation, whereas for hearing participants, this processing mode can be accessed in situations including "interesting" or salient material in the visual periphery. This idea could be addressed in future studies.

\section{Effects of distractor type}

We found delayed responses to target symbols with a face than with an object distractor, but only at the central position. Please note that this effect was unaffected by targetdistractor congruency. The simple co-occurrence with a central face distractor seemed to cause increased filtering costs (Kahneman, Treisman, \& Burkell, 1983). In other words, central faces in the present study were harder to ignore than central objects, but there was no difference in the filtering costs for faces and objects in the visual periphery. The latter result is in contrast to those of Langton, Law, Burton, and Schweinberger (2008), who found stronger filtering costs for faces than for objects in a visual search array located at an eccentricity similar to that of the peripheral distractors in the present study. Unlike the present task, in which the distractor location was to be ignored throughout, participants attended to peripheral distractors, since they had to search the array for a target. Thus, the focus of attention may play a mediating role when explaining those different results.

Another possibility to account for the greater filtering costs caused by central but not peripheral face distractors (as compared to object distractors) is based on the idea that increased filtering costs for central faces are a result of interactive coding of hierarchical cues (eyes vs. face context) in faces, but not in objects (Mohamed, Neumann, \& Schweinberger, 2011). Specifically, eyes are thought to play a central role in face encoding and to be processed by a cluster of eye-sensitive neurons that are separable from, but interact with, face-sensitive neurons in a specific manner (Itier \& Batty, 2009). Because the perception of eyes is severely impaired for unattended faces when they are presented in the visual periphery (Burton, Bindemann, Langton, Schweinberger, \& Jenkins, 2009), it is plausible that peripheral faces were easier to filter out, since the 
normal processes of face encoding may have been less available for peripheral faces.

\section{Summary}

In sum, we found no evidence for an overall group difference in the processing of naturalistic central and peripheral distractor stimuli. Thus, the previously reported enhanced attention in the visual periphery in deaf individuals may be restricted to less meaningful abstract stimuli and may not hold for photographs of faces and objects. However, faces seem to be specifically difficult to filter out when they are presented as central distractors. Most importantly, we found evidence for enhanced attentional capacities in deaf individuals, in that high perceptual load did not abolish distractor processing in this group. This was in striking contrast to the finding of absent distractor processing under high perceptual load for hearing individuals. In future studies, a more fine-grained manipulation of perceptual load may establish more precisely the gradient and boundaries of processing of meaningful and complex distractors in deaf individuals.

\section{References}

Armstrong, B. A., Neville, H. J., Hillyard, S. A., \& Mitchell, T. V. (2002). Auditory deprivation affects processing of motion, but not color. Cognitive Brain Research, 14, 422-434.

Bavelier, D., Brozinsky, C., Tomann, A., Mitchell, T., Neville, H., \& Liu, G. (2001). Impact of early deafness and early exposure to sign language on the cerebral organization for motion processing. Journal of Neuroscience, 21, 8931-8942.

Bavelier, D., Dye, M. W. G., \& Hauser, P. C. (2006). Do deaf individuals see better? Trends in Cognitive Sciences, 10, 512-518.

Bavelier, D., Tomann, A., Hutton, C., Mitchell, T. V., Corina, D., Liu, G., \& Neville, H. (2000). Visual attention to the periphery is enhanced in congenitally deaf individuals. Journal of Neuroscience, 20, RC93.

Beck, D. M., \& Lavie, N. (2005). Look here but ignore what you see: Effects of distractors at fixation. Journal of Experimental Psychology: Human Perception and Performance, 31, 592-607. doi:10.1037/0096-1523.31.3.592

Bottari, D., Nava, E., Ley, P., \& Pavani, F. (2010). Enhanced reactivity to visual stimuli in deaf individuals. Restorative Neurology and Neuroscience, 28, 167-179.

Bottari, D., Turatto, M., Bonfioli, F., Abbadessa, C., Selmi, S., Beltrame, M. A., \& Pavani, F. (2008). Change blindness in profoundly deaf individuals and cochlear implant recipients. Brain Research, 1242, 209-218. doi:10.1016/j.brainres.2008.05.041

Bottari, D., Valsecchi, M., \& Pavani, F. (2012). Prominent reflexive eye-movement orienting associated with deafness. Cognitive Neuroscience, 3, 8-13.

Brebner, J. L., \& Macrae, C. N. (2008). Faces, flowers and football boots: Capacity limits in distractor processing. Cognition, 107, 718-728.

Brozinsky, C. J., \& Bavelier, D. (2004). Motion velocity thresholds in deaf signers: changes in lateralization but not in overall sensitivity. Cognitive Brain Research, 21, 1-10.

Buckley, D., Codina, C., Bhardwaj, P., \& Pascalis, O. (2010). Action video game players and deaf observers have larger Goldmann visual fields. Vision Research, 50, 548-556. doi:10.1016/ j.visres.2009.11.018

Burton, A. M., Bindemann, M., Langton, S. R. H., Schweinberger, S. R., \& Jenkins, R. (2009). Gaze perception requires focused attention: Evidence from an interference task. Journal of Experimental Psychology: Human Perception and Performance, 35, 108-118.

Chen, Q., He, G., Chen, K., Jin, Z., \& Mo, L. (2010). Altered spatial distribution of visual attention in near and far space after early deafness. Neuropsychologia, 48, 2693-2698. doi:10.1016/ j.neuropsychologia.2010.05.016

Chen, Q., Zhang, M., \& Zhou, X. (2006). Effects of spatial distribution of attention during inhibition of return (IOR) on flanker interference in hearing and congenitally deaf people. Brain Research, 1109, 117-127.

Codina, C., Pascalis, O., Mody, C., Toomey, P., Rose, J., Gummer, L., \& Buckley, D. (2011). Visual advantage in deaf adults linked to retinal changes. PLOS ONE, 6, e20417.

Downing, C. J., \& Pinker, S. (1985). The spatial structure of visual attention. In M. I. Posner \& O. S. M. Marin (Eds.), Attention and performance XI: Mechanisms of attention and visual search (pp. 171-187). Hillsdale: Erlbaum.

Dye, M. W. G., Baril, D. E., \& Bavelier, D. (2007). Which aspects of visual attention are changed by deafness? The case of the Attentional Network Test. Neuropsychologia, 45, 1801-1811.

Dye, M. W. G., Hauser, P. C., \& Bavelier, D. (2009). Is visual selective attention in deaf individuals enhanced or deficient? The case of the useful field of view. PLoS ONE, 4, e5640.

Fieger, A., Röder, B., Teder-Sälejärvi, W., Hillyard, S. A., \& Neville, H. J. (2006). Auditory spatial tuning in late-onset blindness in humans. Journal of Cognitive Neuroscience, 18, 149-157.

Finney, E. M., \& Dobkins, K. R. (2001). Visual contrast sensitivity in deaf versus hearing populations: Exploring the perceptual consequences of auditory deprivation and experience with a visual language. Cognitive Brain Research, 11, 171-183.

Itier, R. J., \& Batty, M. (2009). Neural bases of eye and gaze processing: The core of social cognition. Neuroscience and Behavioral Reviews, 33, 843-863.

Kahneman, D., Treisman, A., \& Burkell, J. (1983). The cost of visual filtering. Journal of Experimental Psychology: Human Perception and Performance, 9, 510-522.

Langton, S. R. H., Law, A. S., Burton, A. M., \& Schweinberger, S. R. (2008). Attention capture by faces. Cognition, 107, 330-342.

Lavie, N. (1995). Perceptual load as a necessary condition for selective attention. Journal of Experimental Psychology: Human Perception and Performance, 21, 451-468. doi:10.1037/00961523.21.3.451

Lavie, N. (2005). Distracted and confused? Selective attention under load. Trends in Cognitive Sciences, 9, 75-82. doi:10.1016/ j.tics.2004.12.004

Lavie, N., Ro, T., \& Russell, C. (2003). The role of perceptual load in processing distractor faces. Psychological Science, 14, 510-515.

Loke, W. H., \& Song, S. (1991). Central and peripheral visual processing in hearing and nonhearing individuals. Bulletin of the Psychonomic Society, 29, 437-440.

Minear, M., \& Park, D. C. (2004). A lifespan database of adult facial stimuli. Behavior Research Methods, Instruments, \& Computers, 36, 630-633. doi:10.3758/BF03206543

Mohamed, T., Neumann, M. F., \& Schweinberger, S. R. (2011). Combined effects of attention and inversion on event related potentials to human bodies and faces. Cognitive Neuroscience, 2, 138-146.

Muir, L. J., \& Richardson, I. E. G. (2005). Perception of sign language and its application to visual communications for deaf people. Journal of Deaf Studies and Deaf Education, 10, 390-401.

Neumann, M. F., Mohamed, T. N., \& Schweinberger, S. R. (2011). Face and object encoding under perceptual load: ERP evidence. NeuroImage, 54, 3021-3027. doi:10.1016/j.neuroimage.2010.10.075 
Neumann, M. F., Schweinberger, S. R., Wiese, H., \& Burton, A. M. (2007). Event-related potential correlates of repetition priming for ignored faces. NeuroReport, 18, 1305-1309.

Neville, H. J., \& Lawson, D. (1987a). Attention to central and peripheral visual space in a movement detection task: An event related potential and behavioural study. I: Normal hearing adults. Brain Research, 405, 253-267.

Neville, H. J., \& Lawson, D. (1987b). Attention to central and peripheral visual space in a movement detection task: An event related potential and behavioural study. II: Congenitally deaf adults. Brain Research, 405, 268-283.

Neville, H. J., \& Lawson, D. (1987c). Attention to central and peripheral visual space in a movement detection task: III. Separate effects of auditory deprivation and acquisition of a visual language. Brain Research, 405, 284-294.

Parasnis, I., \& Samar, V. J. (1985). Parafoveal attention in congenitally deaf and hearing young adults. Brain and Cognition, 4, 313-327.

Pavani, F., \& Bottari, D. (2011). Visual abilities in individuals with profound deafness: A critical review. In M. M. Murray \& M. T. Wallace (Eds.), The neural bases of multisensory processes (pp. 423-448). Boca Raton: CRC Press.
Poizner, H., \& Tallal, P. (1987). Temporal processing in deaf signers. Brain and Language, 30, 52-62.

Proksch, J., \& Bavelier, D. (2002). Changes in the spatial distribution of visual attention after early deafness. Journal of Cognitive Neuroscience, 14, 687-701.

Ro, T., Russell, C., \& Lavie, N. (2001). Changing faces: A detection advantage in the flicker paradigm. Psychological Science, 12, 94 99.

Röder, B., Teder-Sälejärvi, W., Sterr, A., Rösler, F., Hillyard, S. A., \& Neville, H. J. (1999). Improved auditory spatial tuning in blind humans. Nature, 400, 162-166.

Rovamo, J., \& Virsu, V. (1979). An estimation and application of the human cortical magnification factor. Experimental Brain Research, 37, 495-510.

Sladen, D. P., Tharpe, A. M., Ashmead, D. H., Grantham, D. W., \& Chun, M. M. (2005). Visual attention in deaf and normal hearing adults: Effects of stimulus compatibility. Journal of Speech, Language and Hearing Research, 48, 1529-1537.

Stevens, C., \& Neville, H. J. (2006). Neuroplasticity as a double-edged sword: Deaf enhancements and dyslexic deficits in motion processing. Journal of Cognitive Neuroscience, 18, 701-714. 